\title{
Chemical Characterization of Cumin Seed Oil (Cuminum Cyminum) by Gcms and its Comparative Study
}

\author{
S. R. Gotmare ${ }^{1}$ and E. A. Tambe ${ }^{2 *}$ \\ ${ }^{1}$ Dept. of Analytical Chemistry, SNDT Women's University, Santacruz, Mumbai, India, 400049 \\ ${ }^{2}$ SNDT Women's University, Santacruz, Mumbai, India, 400049 \\ *Corresponding author: tambees2007@yahoo.com, Mobile no.: - 9819486568
}

Available online at: www.isroset.org

Received: 28/May/2018, Revised: 09/Jun/2018, Accepted: 23/Jun/2018, Online: 30/Jun/ 2018

\begin{abstract}
Cumin (Cuminum cyminum L.) is among the large number of spices used to flavour food and beverages. In this study the chemical composition of the extracted cumin seed oil and market cumin oil were analysed by GCMS and compared. The comparative study revealed that there is a huge difference between these two oils. The extracted cumin oil was found rich in oxygenated terpenoids. In addition to this, a comparative study from the data of chemical composition of cumin seeds from different countries also showed variation in the chemical components depending upon the geography, environment, climate, harvesting stage, etc.
\end{abstract}

Keywords - Hydrodistillation, cuminaldehyde, Iran, antioxidant, antimicrobial

\section{INTRODUCTION}

A spice is substance of plant origin, primarily from various parts of the plant such as dried seed, fruit, root, or bark which is used in very small quantities as a food additive for flavour, colour, or as a preservative. Many spices are also used for purposes of medicine and religious rituals in Asia and in cosmetics, perfumery and liquorices in other parts of the world. Spices are consumed in small quantities, hence the value of spices cannot be judged on their nutritive values, as they do not provide essential nutrients [1]. Cuminum cyminum L. is an annual plant of the family apiaceae, native from the east Mediterranean to east India. The word cumin in English is derived from the Latin cuminum, which itself was derived from Greek "Kyminon" [2]. Cumin seeds resemble caraway seeds but are slightly smaller having oblong shape, thicker in the middle, compressed laterally with nine ridges and yellow-brown in colour. Cumin is the second most popular spice in the world after black pepper. The plant is indigenous to Egypt and Syria and one of the most cultivated popular spices throughout the world [3]. It is mainly grown in India, Syria, Iran and Turkey. Cumin is a characteristic spice of the oriental cuisine and one of the main ingredients of curry powder. In Serbia, cumin is not very popular but recently there has been the increasing interest for healthy nutrition and functional food supplements, as well as traditional remedies approved by modern scientific methods [4]. Cumin seeds contain numerous phyto-chemicals that are known to have antioxidant, carminative and anti-flatulent properties. The active principles in the cumin may increase the motility of the gastro-intestinal tract as well as increase the digestion power by increasing gastro-intestinal enzyme secretions. This spice is an excellent source of minerals like iron, copper, calcium, potassium, manganese, selenium, zinc and magnesium. The seeds are also rich source of many flavonoid phenolic anti-oxidants such as carotenes, zeaxanthin and lutein [5].

The seeds contained a volatile oil mainly composed of monoterpene hydrocarbons, oxygenated mono- and sesquiterpenes, fatty acids, aldehydes, ketones and esters. The cumin seeds are considered as carminative, analgesic, eupeptic, antispasmodic, astringent, used to treat digestive disorders, cough, diarrhoea, dyspepsia, flatulence, morning sickness, colic, dyspeptic headache and bloating and to improve liver function [6]. In herbal medicine, cumin oil is known to possess several pharmacological activities, such as antimicrobial, anti-diabetic, antiepileptic, anti-infertility, anticancerous and immunomodulative effects due to presence of active chemical constituents. The anti-carcinogenic activity has also been studied and cumin seeds are found potent inhibitor of both squamous cell carcomas and hepatomas [7]. The chief constituent of cumin seed oil found in this study is cuminaldehyde along with minor constituents like $\beta$-pinene, $\mathrm{p}$-cymene, $\alpha$-terpinen-7-al and $\gamma$-terpinen-7-al. Cumin seed oil and alcoholic extract inhibited the growth of Klebsiella pneumoniae and its clinical isolates and caused improvement in the cell morphology, capsule expression and decreased urease activity. This property is attributed to cuminaldehyde. A methanolic extract of cumin seeds reduced the blood glucose and inhibited glycosylated haemoglobin, creatinine, blood urea nitrogen and improved serum insulin and glycogen (liver and skeletal muscle) content in alloxan 
and streptozotocin (STZ) diabetic rats [8]. The aim of this study is to understand the chemical composition of cumin seed oil extracted from cumin seeds and to compare the extracted oil composition with the marketed cumin seed oil. In this study a review of variation of chemical composition of cumin seed oil from different countries is also evaluated.

This research paper is divided into 5 parts and these are 1) introduction - in which basic information of cumin seeds is provided, 2) plant description and taxonomy wherein taxonomy of cumin plant is described, 3) methods and materials explains the methodology used in evaluation of chemical constituents of cumin seed oil, 4) results and discussion elucidate the data obtained from GC-MS and lastly $5)$ conclusion of the research work carried out.

\section{PLANT DESCRIPTION AND TAXONOMY}

Cumin is a small, slender, glabrous herbaceous annual of the parsley family, usually reaching $25 \mathrm{~cm}$ (some varieties being double this height) and tend to droop under its own weight.
The blue-green linear leaves are 5-10 cm long, pinnate or bipinnate, thread-like leaflets. The white or pink flowers bloom in small compound umbels. The fruit is a lateral fusiform or ovoid achene 4-5 mm long, containing a single seed [9]. The plants bloom in June and July and are harvested when $85 \%$ of fruits are ripe $[9,10]$. The Unites States Department of Agriculture, Nature Resources Conservation Service provides the taxonomic description of Cumin (Cuminum cyminum) as follows: -
Kingdom: - Plantae - Plants
Subkingdom: - Tracheobionta - Vascular plant
Superdivision: - Spermatophyta - Seed plant
Division: - Magnoliophyta-Flowering plant
Class: - Magnoliopsida-Dicotyledons
Subclass: - Rosidae
Order: - Apiales
Family: - Apiaceae/Umbelliferae - Carrot family
Genus: - Cuminum L. - cumin
Spices: - Cuminum cyminum L. - cumin

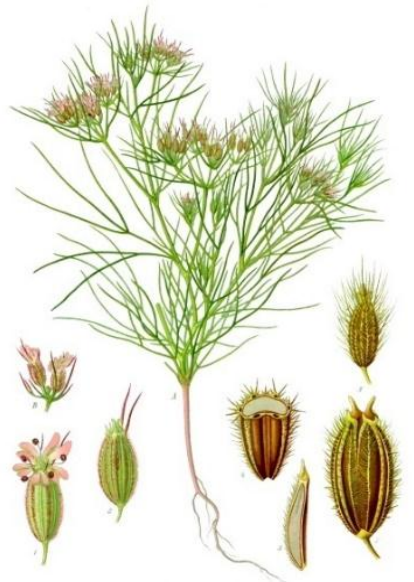

Figure 1a

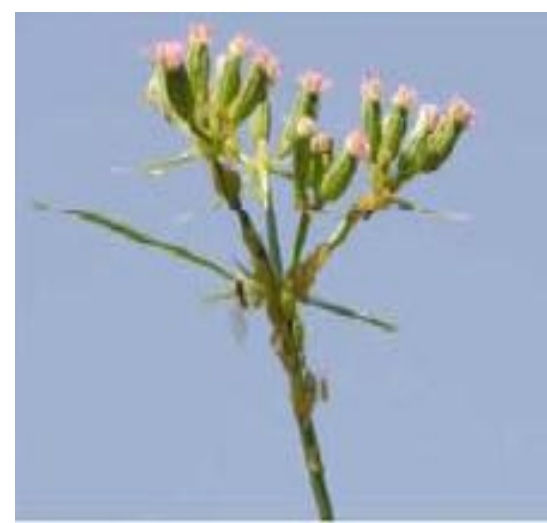

Figure 1b

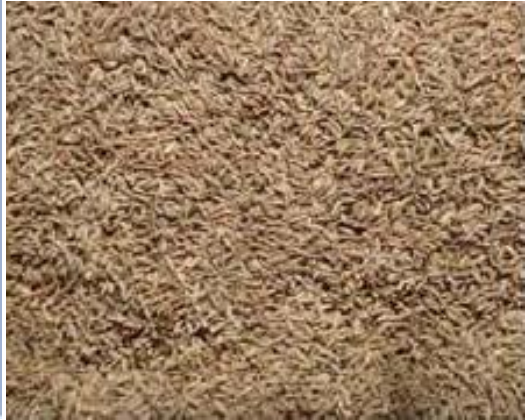

Figure 1c

Figure 1a: - Full grown Cumin plant and seeds. Source: - Internet

Figure 1b: - Picture of flower of cumin plant, figure 1c: - Picture of cumin seeds.

Source: - E. V. Divakara Sastry and Muthuswamy Anandaraj, 2011

\section{MATERIALS AND METHODS}

Plant materials: - The cumin seeds were purchased from D Mart of Thane city, Maharashtra, India and market cumin seed oil was purchased online. The leaflet along with the market cumin oil states 'part of the plant used as seeds'.

Extraction of essential oil: - $125 \mathrm{~g}$ of whole cumin seeds were weighed for extraction of cumin seed essential oil. The weighed cumin seeds were placed in a round bottom flask which was then connected to a distillation condenser. Thus, the cumin seed oil obtained by hydro distillation process in which $700 \mathrm{ml}$ distilled water was used. The round bottom flask was kept in a heating mantle and the temperature was gradually increased from $60^{\circ} \mathrm{C}$ to $80^{\circ} \mathrm{C}, 80^{\circ} \mathrm{C}$ to $90^{\circ} \mathrm{C}$ and finally at $100^{\circ} \mathrm{C}$. The extraction process was carried out for 3 hours (no more oil drop was coming out of the condenser). The oil obtained from whole cumin seeds through extraction process is called as extracted cumin oil. The oil was collected in glass bottle. Both extracted as well as market cumin oil were stored in refrigerator. 


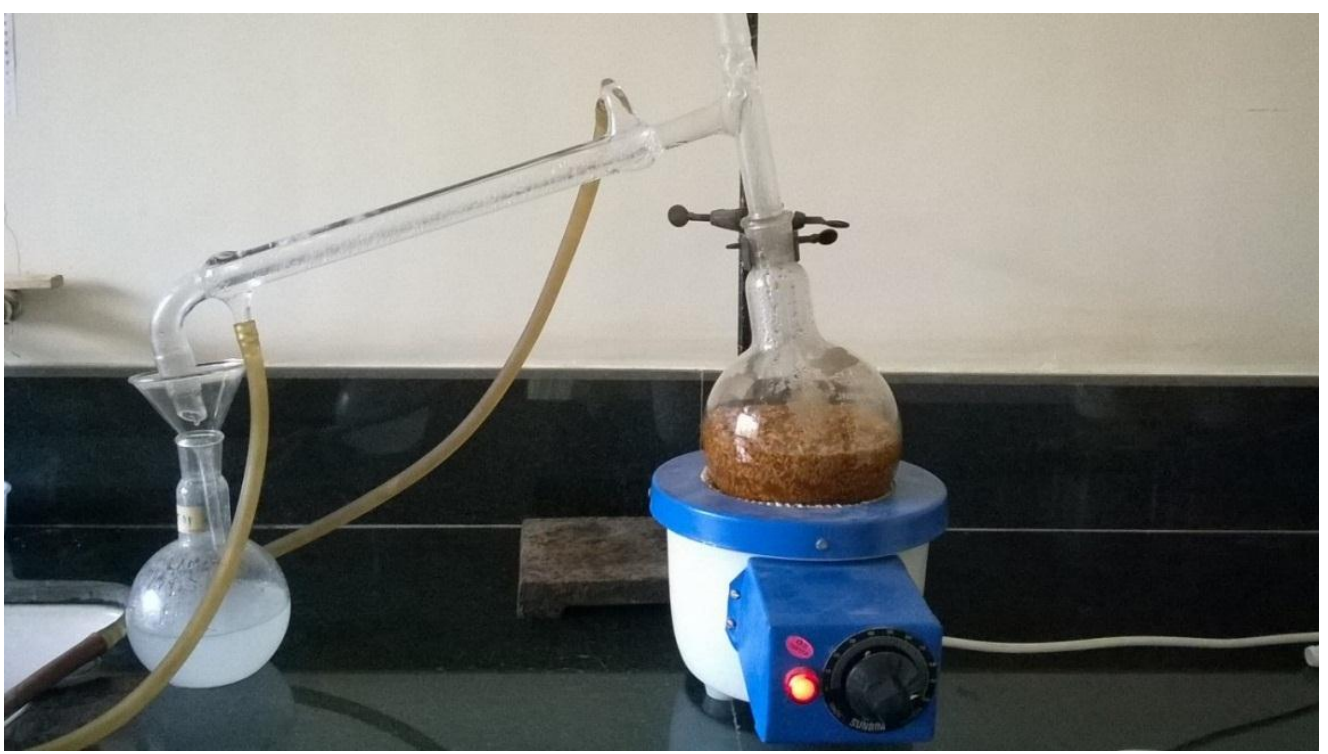

Figure 2 Extraction of whole cumin using distillation flask. Source: - Genuine picture captured at Research lab of S.H.P.T College of Science, SNDT Women's University. PC: - Esha Tambe

GCMS analysis: - The essential oil analysis was carried out on Perkin Elmer Clarus $600 \mathrm{C}$ mass spectrometer. The capillary column used for the analysis was GsBP-5ms with dimensions as $30 \mathrm{~m} \times 0.25 \mathrm{~mm}$ ID, $0.25 \mu \mathrm{m}$ and composed of 5\% Diphenyl and 95\% Dimethylpolysiloxane (nonpolar column) with temperature range of $-60^{\circ} \mathrm{C}$ to $350^{\circ} \mathrm{C}$. Helium $(\mathrm{He})$ was used as a carrier gas with the flow rate of 1 $\mathrm{ml} / \mathrm{min} .0 .2 \mu \mathrm{l}$ of the essential oil samples were injected as it is with a split ratio of 50:1 so as to avoid overloading of the column. The oven temperature was programmed as $60^{\circ} \mathrm{C}$ hold for 2 mins initially then $60^{\circ} \mathrm{C}$ to $120^{\circ} \mathrm{C}$ at the rate of 5 degree/min and kept hold for 0 mins at $120^{\circ} \mathrm{C}$ followed by $120^{\circ} \mathrm{C}$ to $260^{\circ} \mathrm{C}$ at the rate of 5 degree $/ \mathrm{min}$. The total run time was 42 minutes. The injector was kept at $220^{\circ} \mathrm{C}$ and MS source and Inlet line temperature was kept at $280^{\circ} \mathrm{C}$. The mass range was kept from 15 to $350 \mathrm{amu}$ which is sufficient for organic compounds.

\section{RESULTS AND DISCUSSIONS}

Extraction yield: - The extracted cumin oil has bright yellow colour while the market cumin oil has dark yellow colour. $125 \mathrm{~g}$ of cumin seeds yielded $1.43 \mathrm{~g}$ of cumin seed oil. The percentage yield of essential oil was calculated as per Shimaa A. Moawad et al, 2015 [11]. The percentage yield is calculated on the weight basis. The equation is as follows: -

Volatile oil $(\%)=($ Weight of the volatile oil recovered in $\mathrm{g}$ $\mathrm{x}$ 100)/ Weight of sample taken in $\mathrm{g}$

The percentage yield for the extracted cumin oil was found to be $1.14 \%$.

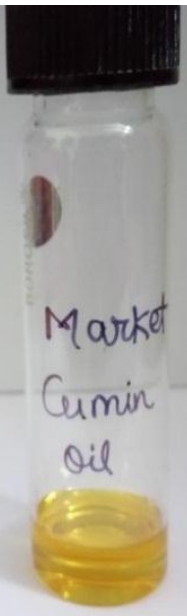

Figure 3 Extracted cumin oil and Market cumin oil genuine pictures. PC: - Esha Tambe 
Chemical composition of extracted cumin oil and market cumin oil under study: - The identification of chemical composition of extracted as well as market cumin oil was performed using GCMS. The Table 1 shows the compounds identified by GCMS present in cumin oils.

\begin{tabular}{|c|c|c|c|c|}
\hline $\begin{array}{l}\text { Sr. } \\
\text { no. }\end{array}$ & Name of the compound & RT & $\begin{array}{l}\text { Cumin oil extracted from } \\
\text { whole seeds }(\%)\end{array}$ & $\begin{array}{l}\text { Market cumin } \\
\text { oil }(\%)\end{array}$ \\
\hline 1 & $\alpha$-Pinene & 6.119 & -- & 0.15 \\
\hline 2 & $\beta$-Pinene & 7.22 & 1.58 & 1.63 \\
\hline 3 & p-cymene & 8.521 & 6.67 & 17.36 \\
\hline 4 & Limonene & 8.631 & $\begin{array}{ll}-- \\
\end{array}$ & 0.19 \\
\hline 5 & Eucalyptol; 1,8-cineole & 8.675 & 0.46 & -- \\
\hline 6 & $\gamma$-Terpinene & 9.414 & 0.36 & 3.51 \\
\hline 7 & p-Cymenene & 10.269 & $\begin{array}{ll}-- \\
\end{array}$ & 0.47 \\
\hline 8 & 1-Butyl cyclohexene & 11.993 & 0.15 & -- \\
\hline 9 & $\begin{array}{l}\text { trans-p-Mentha-2,8-dien-1-ol; 1-Methyl-4-(1- } \\
\text { methylethenyl)-2-cyclohexen-1-ol }\end{array}$ & 12.487 & -- & 0.17 \\
\hline 10 & $\begin{array}{l}\text { (-)-terpinen-4-ol; 4-methyl-1-(1-methylethyl-)- } \\
\text { 3-cyclohexen-1-ol }\end{array}$ & 12.778 & -- & 0.20 \\
\hline 11 & 4'-Methyl acetophenone & 13.012 & -- & 0.20 \\
\hline 12 & $\alpha$-Terpineol & 13.159 & -- & 0.29 \\
\hline 13 & p-Menth-3-en-7-al & 13.258 & 1.11 & -- \\
\hline 14 & $\begin{array}{lll}(+)- & \text { Dihydrocarvone, } & \text { 2-Methyl-5-(1- } \\
\text { methylethenyl)-cyclohexanone } & \end{array}$ & 13.334 & -- & 0.27 \\
\hline 15 & Cuminaldehyde & 14.958 & 74.62 & 64.31 \\
\hline 16 & $\begin{array}{l}\text { Phellandral, } \\
\text { methylethyl)-1-Cyclohexene }\end{array}$ & 15.559 & 0.14 & 0.30 \\
\hline 17 & $\alpha$-Terpinen-7-al & 15.881 & 3.98 & 4.59 \\
\hline 18 & o-Cymen-7-ol & 15.965 & --- & 1.11 \\
\hline 19 & $\gamma$-Terpinen-7-al & 16.072 & 7.95 & -- \\
\hline 20 & Thymol & 16.186 & $\begin{array}{ll}-- \\
\end{array}$ & 0.35 \\
\hline 21 & $\begin{array}{l}\text { 3-Cyclohexene-1-carboxaldehyde, } 1,3,4- \\
\text { trimethyl- }\end{array}$ & 16.493 & -- & 0.28 \\
\hline 22 & 4-Hydroxy-cryptone & 16.797 & 1.68 & 0.38 \\
\hline 23 & Cumic acid, 4-(1-methylethyl)-benzoic acid & 20.047 & 0.97 & 1.43 \\
\hline 24 & $\begin{array}{l}\beta \text {-Farnesene;1,6,10-Dodecatriene, } 7,11 \\
\text { Dimethyl-3-methylene }\end{array}$ & 20.171 & -- & 0.17 \\
\hline 25 & $\alpha$-Neocallitropsene & 20.741 & -- & 2.19 \\
\hline \multicolumn{5}{|c|}{ Chemical classes } \\
\hline \multicolumn{3}{|c|}{ Aliphatic hydrocarbons } & -- & 0.17 \\
\hline \multicolumn{3}{|c|}{ Monoterpene hydrocarbons } & 2.09 & 7.67 \\
\hline \multicolumn{3}{|c|}{ Oxygenated terpenoids } & 90.91 & 71.17 \\
\hline \multicolumn{3}{|c|}{ Aromatic hydrocarbons } & 6.67 & 20.37 \\
\hline
\end{tabular}

RT - Retention time, (--) - not found

Table no. 1 chemical composition of extracted cumin oil and market cumin oil

The compounds present in cumin oil were broadly classified into four classes as: - aliphatic hydrocarbons, monoterpene hydrocarbons, oxygenated terpenoids and aromatic hydrocarbons. The cumin oil extracted from whole cumin using distillation flask constitute high amount of oxygen containing terpene derivatives. Aliphatic hydrocarbons were not found while monoterpenes and aromatic hydrocarbons were not present more than $10 \%$. The market cumin oil also showed presence of good amount of oxygenated terpenoids but with a difference of about $19.74 \%$ with that of extracted cumin oil. Aromatic hydrocarbons $(20.37 \%)$ were found to be second highest class of organic compounds present in market cumin oil. The difference between the percentage amounts of aromatic hydrocarbons between market and extracted cumin oil is significant $(13.7 \%)$.

Cuminaldehyde was found to be present as a major oxygenated terpenoid in both extracted and market cumin 
oils. But the concentration of cuminaldehyde was much higher in extracted cumin oil $(74.62 \%)$ than in market cumin oil $(64.31 \%)$. The market oil contained $10.31 \%$ less amount of cuminaldehyde. However, p-cymene, a natural antioxidant, was found in good amount in market oil (17.36\%) while extracted cumin oil contained only $6.67 \%$. The concentration of p-cymene was $10.69 \%$ greater in market oil. Extracted cumin oil constitute of an antimicrobial agent, $\gamma$-Terpinen-7al, up to $8 \%$. $\alpha$-Terpinen-7-al, another antimicrobial agent, was found proportionately in extracted and market cumin oils. A minor difference of about $0.61 \%$ was found in the concentration of $\alpha$-Terpinen-7-al between market and extracted cumin oil. Eucalyptol, p-Menth-3-en-7-al and 1Butyl cyclohexene were present only in extracted cumin oil. Likewise, market cumin oil showed presence of compounds namely, p-Cymenene, trans-p-Mentha-2,8-dien-1-ol, terpinen4-ol, 4-methyl acetophenone, $\alpha$-Terpineol, (+)Dihydrocarvone, Thymol, 3-Cyclohexene-1-carboxaldehyde, 1,3,4-trimethyl-, $\beta$-Farnesene and $\alpha$-Neocallitropsene but their concentrations were not more than $0.50 \%$; except for $\alpha$ Neocallitropsene. $\quad \beta$-Farnesene is the only aliphatic sesquiterpene which became a part of market cumin oil. $\gamma$ Terpinene contributed to market cumin oil composition. Thus, the extracted and market cumin oil showed a significant contrast in the chemical composition.

The main components of extracted cumin oil were cuminaldehyde $(74.62 \%), \gamma$-Terpinen-7-al (7.95\%), p-cymene (6.67\%), $\alpha$-Terpinen-7-al (3.98\%), 4-Hydroxy cryptone (1.68), $\beta$-pinene (1.58) and p-Menth-3-en-7-al (1.11\%). Whereas the main components of market cumin oil were cuminaldehyde $(64.31 \%)$, p-cymene $(17.36 \%)$, $\alpha$-Terpinen-7al $(4.59 \%), \gamma$-Terpinene $(3.51 \%), \alpha$-Neocallitropsene $(2.19 \%)$, $\beta$-pinene $(1.63 \%)$, Cumic acid $(1.43 \%)$ and o-Cymen-7-ol $(1.11 \%)$.

The figure 4 and figure 5 are the chromatograms of the extracted cumin oil and market cumin oil respectively.

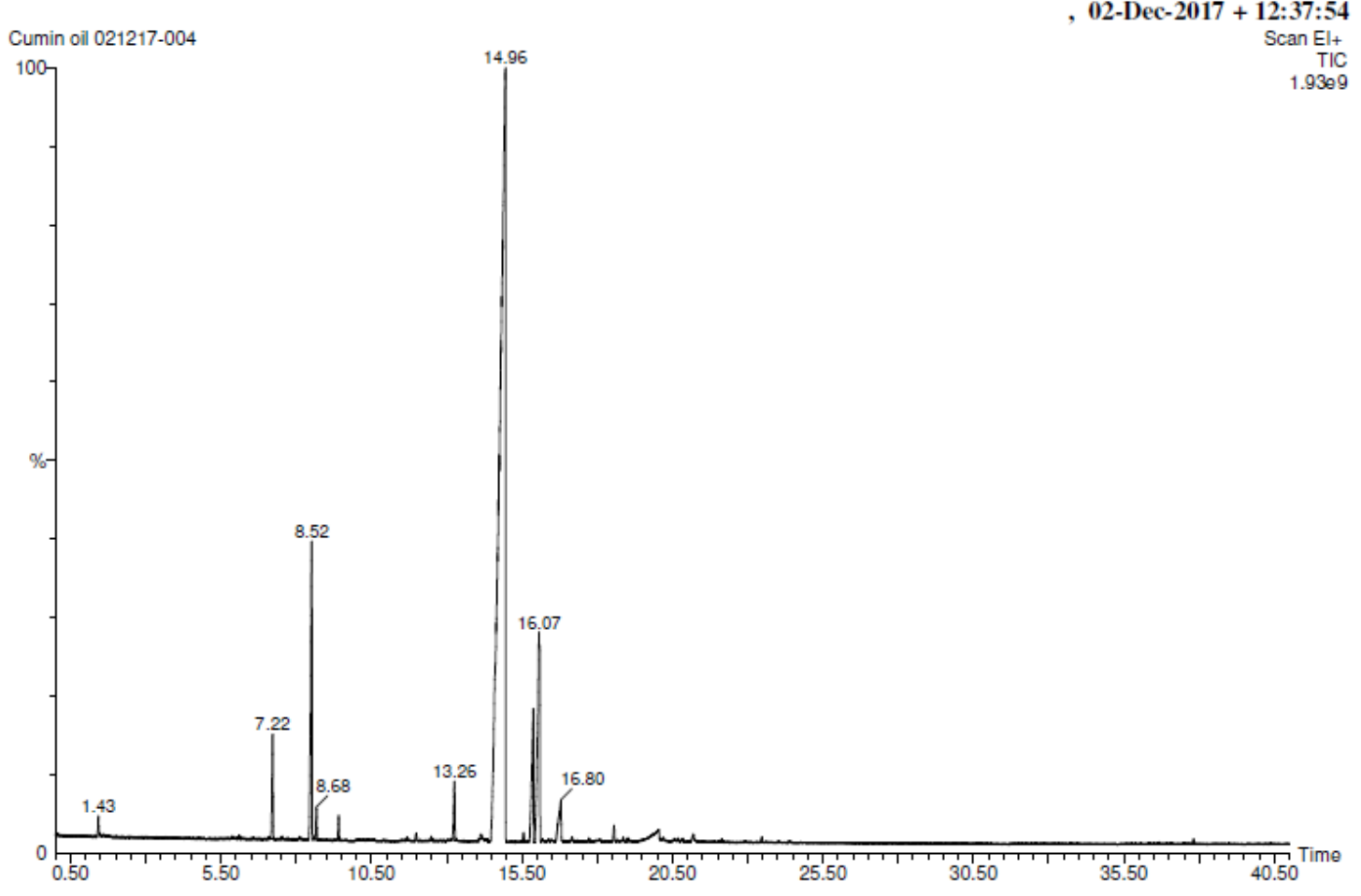

Figure 4 chromatogram of extracted cumin oil. 


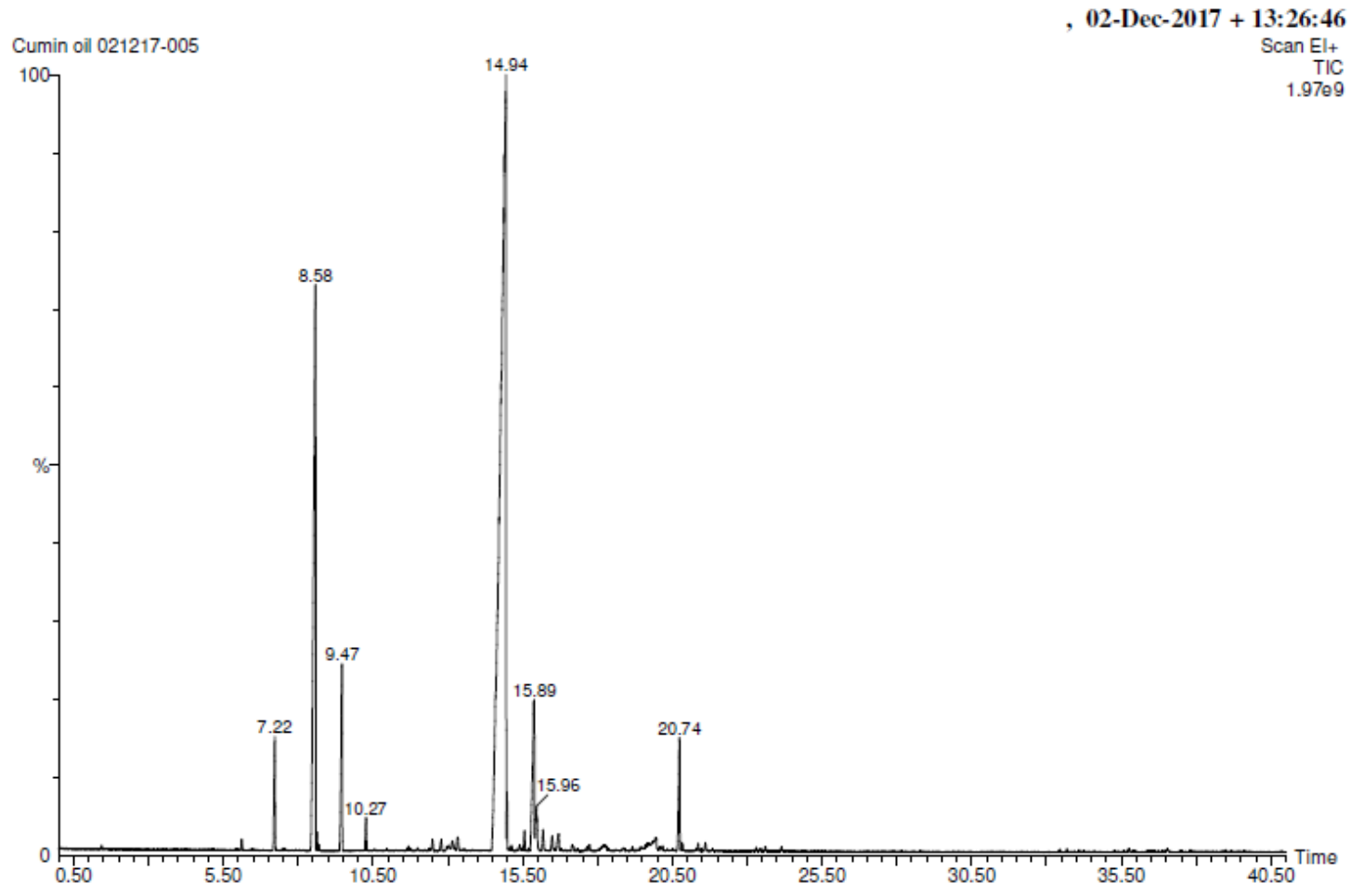

Figure 5 chromatogram of market cumin oil

Comparative study of chemical composition of cumin oil data from seven different countries: - The data available from literature review was compiled to understand the chemical composition of the cumin oil. These cumin oils were extracted from cumin seeds grown in nine different countries.

\begin{tabular}{|c|c|c|c|}
\hline $\begin{array}{l}\text { Sr. } \\
\text { no. }\end{array}$ & Components and their percentages & Origin & Reference \\
\hline 1 & $\begin{array}{l}\text { Thymol }(0.495 \%) \text {, Anisyl alcohol }(1.934 \%) \text {, Cuminaldehyde }(14.279 \%) \text {, Vanillin } \\
(2.769 \%) \text {, Benzoic acid }(0.214 \%)\end{array}$ & Iraq & $\begin{array}{l}\text { Fanar Hashum } \\
\text { Yousif Al-Hashemi } \\
\text { [2] }\end{array}$ \\
\hline 2 & $\begin{array}{l}\text { Isobutyl isobutyrate }(0.8 \%), \alpha \text {-Pinene }(29.1 \%) \text {, Sabinene }(0.6 \%) \text {, Limonene }(21.5 \%), 1,8- \\
\text { Cineole }(17.9 \%), \gamma \text {-Terpinene }(0.6 \%) \text {, Linalool }(10.4 \%), \alpha \text {-Terpineole }(3.17 \%) \text {, Geraniol } \\
(1.1 \%) \text {, Linalyl acetate }(4.8 \%), \alpha \text {-Terpinyl acetate }(1.3 \%) \text {, Methyl eugenol }(1.6 \%)\end{array}$ & Iran & $\begin{array}{l}\text { Latif Gachkar et al } \\
\qquad[12]\end{array}$ \\
\hline 3 & $\begin{array}{l}\alpha \text {-phellandrene }(0.82 \%) \text {, Limonene }(0.5 \%),(\mathrm{E})-\beta \text {-ocimene }(9.72 \%), \gamma \text {-terpenine }(12 \%), \alpha- \\
\text { terpinolene }(3 \%), \mathrm{p} \text {-menth-2-en-1-ol }(9 \%), \text { Cuminldehyde }(35.25 \%) \text {, Geraniol }(0.63 \%), 2- \\
\text { caren-10-al }(1.32 \%), \alpha \text {-Terpinyl acetate }(5.32 \%) \text {, Tetradecene }<1->(12.25 \%) \text {, Manoyl } \\
\text { oxide }(0.63 \%), \text { Phynyl ethyl anthranilate-2- }(4.70 \%)\end{array}$ & Egypt & $\begin{array}{l}\text { Shimaa A. Moawad } \\
\text { [11] }\end{array}$ \\
\hline 4 & $\begin{array}{l}\text { Isobutyl isobutyrate }(0.8 \%), \alpha \text {-Pinene }(29.2 \%) \text {, Sabinene }(0.6 \%) \text {, Limonene }(21.7 \%), 1,8 \text { - } \\
\text { Cineole }(18.1 \%), \gamma \text {-Terpinene }(0.6 \%) \text {, Linalool }(10.5 \%) \text {, } \\
\alpha \text {-Terpineole }(3.17 \%) \text {, Geraniol }(1.1 \%) \text {, Linalyl acetate }(4.8 \%), \alpha \text {-Terpinyl acetate }(1.3 \%) \text {, } \\
\text { Methyl eugenol }(1.6 \%)\end{array}$ & Iran & $\begin{array}{l}\text { Hossein } \\
\text { Mohammadpour et al } \\
\text { [13] }\end{array}$ \\
\hline 5 & $\begin{array}{l}\alpha \text {-Pinene }(30 \%), \text { Limonene }(21 \%), 1,8 \text {-Cineole }(18.5 \%), \text { Linalool }(10 \%), \text { Linalyl acetate } \\
(4 \%), \alpha \text {-Terpineol }(3 \%), \alpha \text {-Terpineol acetate }(1.5 \%), \text { Geraniol }(1.5 \%), \text { Methyl eugenol } \\
(1.2 \%), \text { Sabinene }(0.5 \%), \text { Terpinen-4-ol }(0.5 \%), \\
\text { Terpinolene }(0.5 \%), \gamma \text {-terpinene }(0.5 \%)\end{array}$ & Iran & $\begin{array}{l}\text { A. Naeini } \\
\text { and H. Shokri [14] }\end{array}$ \\
\hline 6 & $\begin{array}{l}\alpha \text {-Pinene }(0.5 \%), \beta \text {-Pinene }(7.8 \%) \text {, Myrcene }(0.6 \%), p \text {-Cymene }(9.9 \%) \text {, Limonene }(0.5 \%) \text {, } \\
\gamma \text {-Terpinene }(11.1 \%) \text {, Terpineol-4 }(0.6 \%) \text {, Cuminaldehyde }(36.3 \%) \text {, Safranal }(10.9 \%),\end{array}$ & China & $\begin{array}{l}\text { Rong Li and Zi-Tao } \\
\text { Jiang [15] }\end{array}$ \\
\hline
\end{tabular}




\begin{tabular}{|c|c|c|c|}
\hline & Cuminic alcohol $(16.9 \%)$ & & \\
\hline \multirow{11}{*}{7} & $\begin{array}{l}\beta \text {-Pinene }(9.25 \%), \text { Myrcene }(0.87 \%), \alpha \text {-Phellandrene }(1.86 \%), \mathrm{p} \text {-Cymene }(8.84 \%), \gamma \text { - } \\
\text { Terpinene }(19.59 \%), \alpha \text {-Pinene oxide }(0.79 \%) \text { Cuminaldehyde }(21.83 \%), \text { Safranal } \\
(20.21 \%), \gamma \text {-Terpinene- } 7 \text {-al }(14.25 \%), \alpha \text {-Cedrene }(0.65 \%)\end{array}$ & $\begin{array}{l}\text { Esfarayen } \\
\text { (Iran) }\end{array}$ & \multirow{11}{*}{$\begin{array}{c}\text { Neda Hashemian et al } \\
\text { [16] }\end{array}$} \\
\hline & $\begin{array}{l}\beta \text {-Pinene }(7.87 \%), \text { Myrcene }(0.90 \%), \alpha \text {-Phellandrene }(1.17 \%) \text {, p-cymene }(6.96 \%), \gamma \text { - } \\
\text { Terpinene }(17.22 \%), \alpha \text {-Pinene oxide }(1.41 \%) \text {, Cuminaldehyde }(20.50 \%) \text {, Safranal } \\
(24.89 \%), \gamma \text {-Terpinene-7-al }(16.13 \%),\end{array}$ & $\begin{array}{l}\text { Ghayen } \\
\text { (Iran) }\end{array}$ & \\
\hline & $\begin{array}{l}\beta \text {-Pinene }(8.65 \%), \text { Myrcene }(1.01 \%), \alpha \text {-Phellandrene }(1.20 \%), \text { p-cymene }(6.69 \%), \gamma- \\
\text { Terpinene }(19.41 \%), \alpha \text {-Pinene oxide }(1.05 \%) \text {, Cuminaldehyde }(20.63 \%), \text { Safranal } \\
(24.89 \%), \gamma \text {-Terpinene-7-al }(13.76 \%),\end{array}$ & $\begin{array}{l}\text { Boshroyeh } \\
\text { (Iran) }\end{array}$ & \\
\hline & $\begin{array}{l}\beta \text {-Pinene }(8.32 \%), \text { Myrcene }(0.88 \%), \alpha \text {-Phellandrene }(1.09 \%), \text { p-cymene }(7.69 \%), \gamma- \\
\text { Terpinene }(18.33 \%), \alpha \text {-Pinene oxide }(1.18 \%) \text {, Cuminaldehyde }(20.54 \%) \text {, Safranal } \\
(20.94 \%), \gamma \text {-Terpinene-7-al }(19.40 \%)\end{array}$ & $\begin{array}{l}\text { Sarayan } \\
\text { (Iran) }\end{array}$ & \\
\hline & $\begin{array}{l}\text {-Pinene }(7.61 \%), \text { Myrcene }(0.87 \%), \alpha \text {-Phellandrene }(1.19 \%), \mathrm{p} \text {-cymene }(4.14 \%), \beta \text { - } \\
\text { Phellandrene }(0.54 \%), \gamma \text {-Terpinene }(15.00 \%), \alpha \text {-Pinene oxide }(1.35 \%), \text { Cuminaldehyde } \\
(20.09 \%), \text { Safranal }(28.97 \%), \gamma \text {-Terpinene-7-al }(16.69 \%)\end{array}$ & $\begin{array}{l}\text { Ferdos } \\
\text { (Iran) }\end{array}$ & \\
\hline & $\begin{array}{l}\beta \text {-Pinene }(7.19 \%), \text { Myrcene }(0.81 \%), \alpha \text {-Phellandrene }(1.18 \%), p \text {-cymene }(7.80 \%), \gamma- \\
\text { Terpinene }(16.49 \%), \alpha \text {-Pinene oxide }(0.87 \%), \text { Cuminaldehyde }(22.29 \%), \text { Safranal } \\
(25.85 \%), \gamma \text {-Terpinene-7-al }(15.74 \%)\end{array}$ & $\begin{array}{l}\text { Nayshaboor } \\
\text { (Iran) }\end{array}$ & \\
\hline & $\begin{array}{l}\beta \text {-Pinene }(7.15 \%), \text { Myrcene }(0.86 \%), \alpha \text {-Phellandrene }(1.27 \%), p \text {-cymene }(4.89 \%), \beta- \\
\text { Phellandrene }(0.82 \%), \gamma \text {-Terpinene }(15.39 \%), \alpha \text {-Pinene oxide }(1.22 \%), \text { Cuminaldehyde } \\
(20.43 \%), \text { Safranal }(26.58 \%), \gamma \text {-Terpinene- } 7 \text {-al }(18.65 \%)\end{array}$ & $\begin{array}{l}\text { Bardeskan } \\
\quad \text { (Iran) }\end{array}$ & \\
\hline & $\begin{array}{l}\beta \text {-Pinene }(6.79 \%), \text { Myrcene }(0.95 \%), \alpha \text {-Phellandrene }(1.49 \%), \mathrm{p} \text {-cymene }(6.79 \%), \gamma- \\
\text { Terpinene }(16.89 \%), \alpha \text {-Pinene oxide }(1.58 \%), \text { Cuminaldehyde }(20.44 \%), \text { Safranal } \\
(16.83 \%), \gamma \text {-Terpinene-7-al }(25.47 \%)\end{array}$ & $\begin{array}{l}\text { Mahvalat } \\
\text { (Iran) }\end{array}$ & \\
\hline & $\begin{array}{l}\beta \text {-Pinene }(9.62 \%), \text { Mrycene }(0.73 \%), \alpha \text {-Phellandrene }(1.17 \%), \mathrm{p} \text {-cymene }(5.02 \%), \beta- \\
\text { Phellandrene }(0.54 \%), \gamma \text {-Terpinene }(14.07 \%), \alpha \text {-Pinene oxide }(0.83 \%), \text { Cuminaldehyde } \\
(21.62 \%), \text { Safranal }(25.27 \%), \gamma \text {-Terpinene- } 7 \text {-al }(20.83 \%)\end{array}$ & $\begin{array}{l}\text { Sabzevar } \\
\text { (Iran) }\end{array}$ & \\
\hline & $\begin{array}{l}\beta \text {-Pinene }(10.39 \%), \text { Myrcene }(1.17 \%), \alpha \text {-Phellandrene }(0.98 \%), p-c y m e n e(6.60 \%), \gamma- \\
\text { Terpinene }(19.37 \%), \alpha \text {-Pinene oxide }(1.39 \%), \text { Cuminaldehyde }(17.54 \%), \text { Safranal } \\
(16.82 \%), \gamma \text {-Terpinene-7-al }(22.23 \%)\end{array}$ & $\begin{array}{l}\text { Bajestan } \\
\text { (Iran) }\end{array}$ & \\
\hline & $\begin{array}{l}\beta \text {-Pinene }(9.89 \%), \text { Myrcene }(1.02 \%), \alpha \text {-Phellandrene }(1.64 \%), \text { p-cymene }(5.64 \%), \gamma- \\
\text { Terpinene }(18.02 \%), \alpha \text {-Pinene oxide }(1.36 \%), \text { Cuminaldehyde }(20.25 \%), \text { Safranal } \\
(26.78 \%), \gamma \text {-Terpinene-7-al }(13.52 \%)\end{array}$ & $\begin{array}{l}\text { Gonabad } \\
\text { (Iran) }\end{array}$ & \\
\hline 8 & $\begin{array}{l}\alpha \text {-Thujene }(1.45 \%), \alpha \text {-Pinene }(3.47 \%), \text { Camphene }(2.31 \%), \beta \text {-Pinene }(5.38 \%), \text { Myrcene } \\
(1.07 \%), \alpha \text {-Phellandrene }(0.94 \%), \text { Limonene }(1.04 \%), \gamma \text {-Terpinene }(24.51 \%) \text {, Terpinen-4- } \\
\text { ol }(2.00 \%), \text { Acetoxylinalool }(0.57 \%), \alpha \text {-Terpinolene }(1.17 \%) \text {, Cuminaldehye }(1.79 \%), \\
\text { Thymol }(40.68 \%)\end{array}$ & Iran & $\begin{array}{l}\text { Ali Reza Ladan } \\
\text { Moghadam [17] }\end{array}$ \\
\hline 9 & $\begin{array}{l}\beta \text {-Pinene }(1.23 \%), \mathrm{p} \text {-cymene }(5.34 \%), \gamma \text {-Terpinene }(13.96 \%), \text { Transe-4-caranone }(0.58 \%), \\
\text { Cuminaldehyde }(41.56 \%), \alpha \text {-Terpinene-7-al }(12.58 \%), \gamma \text {-Terpinene- } 7 \text {-al }(17.14 \%), \\
\text { Daucene }(0.58 \%), \text { cis- } \beta \text {-Farnesene }(0.58 \%), 10 \text {-epi- } \beta \text {-Acoradiene }(2.01 \%), \text { Caratol }(0.78 \%)\end{array}$ & Iran & $\begin{array}{l}\text { D. Morshedi et al } \\
{[18]}\end{array}$ \\
\hline 10 & $\begin{array}{l}\text { Sabinene }(4.74 \%), \beta \text {-Pinene }(0.76 \%), \mathrm{n} \text {-Decane }(1.24 \%), \gamma \text {-Terpinene }(4.76 \%), 1,8- \\
\text { Cineole }(9.40 \%), \text { n-Dodecane }(25.54 \%), \text { Cuminaldehyde }(14.56 \%), \alpha \text {-Terpinene- }- \text {-al } \\
(33.48 \%), \text { Carvacrol }(0.54 \%)\end{array}$ & Iran & $\begin{array}{l}\text { Zinab Ghafari et al } \\
{[19]}\end{array}$ \\
\hline 11 & $\begin{array}{l}\beta \text {-Pinene }(16.2876 \%), \alpha \text {-Terpinene }(5.6551), \mathrm{p} \text {-cymene }(15.5462 \%), 1,8 \text {-Cineole } \\
(2.2421 \%), \text { Cuminaldehyde }(25.8749 \%), \text { Cuminyl alcohol }(30.023 \%)\end{array}$ & Pakistan & $\begin{array}{l}\text { Asif Hanif Chaudhry } \\
\text { et al [20] }\end{array}$ \\
\hline 12 & $\begin{array}{l}\alpha \text {-Pinene }(29.1 \%), \text { Sabinene }(0.6 \%), \text { Limonene }(21.5 \%), 1,8 \text {-Cineole }(17.9 \%), \gamma \text {-Terpinene } \\
(0.6 \%), \text { Linalool }(10.4 \%), \alpha \text {-Terpineole }(3.17 \%), \text { Geraniol }(1.1 \%), \text { Linalyl acetate }(4.8 \%), \\
\alpha \text {-Terpinyl acetate }(1.3 \%) \text {, Methyl eugenol }(1.6 \%),\end{array}$ & Iran & $\begin{array}{c}\text { Ahmad Rafiee Pour } \\
\text { et al [21] }\end{array}$ \\
\hline 13 & $\begin{array}{l}\alpha \text {-Pinene }(1.18 \%), \beta \text {-Pinene }(12.06 \%), \mathrm{p} \text {-cymene }(6.43 \%), \gamma \text {-Terpinene }(16.58 \%), \mathrm{p}- \\
\text { Menthadien-7-ol }(1,3-C y c l o h e x a d i e n e-1-\text {-methanol,4-(1-methylethyl) }(0.94 \%), \\
\text { Cuminaldehyde (Benzaldehyde,4-(1-methylethyl) }(28.55 \%), 2 \text {-Caren-10-al }(29.64 \%), 3,4- \\
\text { Dimethyl-2-oxocyclopent-3-enylacetic acid }(0.62 \%)\end{array}$ & Sudan & $\begin{array}{c}\text { Muna F. Abushama } \\
\text { et al [22] }\end{array}$ \\
\hline
\end{tabular}

\section{Table no. 2 chemical composition of cumin seeds from seven different countries}

Fanar Hashum Yousif Al-Hashemi showed in his study that the unripe fruit contained Benzoic acid (1.652\%) while a fully ripe cumin seed constitutes of $14.279 \%$ Cuminaldehyde, $2.769 \%$ Vanillin and $1.934 \%$ Anisyl alcohol. Latif Gachkar et al, Hossein Mohammadpour et al, A. Naeini and H. Shokri and Ahmad Rafiee Pour et al found Limonene, 1, 8-Cineole,
Linalool was found in more than $10 \%$ while $\alpha$-Terpineole, geraniol, linalyl acetate, $\alpha$-Terpinyl acetate and methyl eugenol were found in the range of $1-5 \%$. Cuminaldehye was not reported to be found. The areas from where these samples were collected lies to the north region of Iran. All these areas found to be near to the Caspian Sea. Neda 
Hashemian et al reported main constituents of Cuminum cyminum as Cuminaldehyde, Safranal, $\gamma$-Terpinene, $\gamma$ Terpinene-7-al, p-cymene and $\alpha$-Pinene oxide. The cumin seeds were harvested from 11 regions of Khorasan Provinces which located to the northeast of Iran. Ali Reza Ladan Moghadam collected samples from Kurdistan Mountain, Iran (located to west of Iran) which showed presence of Thymol and $\gamma$-Terpinene as major components of cumin essential oil. While $\alpha$-Thujene, $\alpha$-Pinene, Camphene, $\beta$-Pinene, Myrcene, cuminaldehyde and Terpinen-4-ol were found in less than $10 \%$. Zinab Ghafari et al reported presence of aliphatic hydrocarbon $\mathrm{n}$-Dodecane $(25.54 \%)$ as second major compound and terpenes such as $\alpha$-Terpinene-7-al (33.48\%) ranked first followed by cuminaldehyde $(14.56 \%)$ and $\gamma$ Terpinene $(4.76 \%)$. The location from where the samples were collected were not mentioned.

Cumin oil extracted from cumin seeds collected from Mosul city, Iraq reported cuminaldehyde and vanillin as its major components. Egypt's cumin oil reported presence of cuminaldehyde, $\gamma$-terpenine, 1-Tetradecene, (E)- $\beta$-ocimene, p-menth-2-en-1-ol, $\alpha$-Terpinyl acetate, $\alpha$-terpinolene, Phynyl ethyl anthranilate-2- and 2-caren-10-al. Rong Li and Zi-Tao

\begin{tabular}{|c|c|c|c|c|c|c|c|c|}
\hline $\begin{array}{l}\text { Sr. } \\
\text { no. }\end{array}$ & Name of the compound & $\begin{array}{l}\text { New Delhi* } \\
\text { (Nisha } \\
\text { Chaudhary et } \\
\text { al) [3] }\end{array}$ & $\begin{array}{l}\text { Cumin } 1 * * \\
\text { (Milica G. } \\
\text { Aćimović et } \\
\text { al) [4] }\end{array}$ & $\begin{array}{l}\text { Cumin 2** } \\
\text { (Milica G. } \\
\text { Aćimović et } \\
\text { al) [4] }\end{array}$ & $\begin{array}{l}\text { Cumin } 3 * * \\
\text { (Milica G. } \\
\text { Aćimović et } \\
\text { al) [4] }\end{array}$ & $\begin{array}{c}\text { Cumin } 4 * * \\
\text { (Milica G. } \\
\text { Aćimović et al) } \\
{[4]}\end{array}$ & $\begin{array}{l}\text { Gujarat* } \\
\text { (Virendra } \\
\text { Rana) [23] }\end{array}$ & $\begin{array}{l}\text { Amravati* } \\
\text { (Sahadeo Patil } \\
\text { et al) }[24]\end{array}$ \\
\hline 1 & $\alpha$-Pinene & 1.78 & 1.2 & 0.8 & 0.7 & 0.9 & $\mathrm{nmt}$ & 1.41 \\
\hline 2 & Camphene & 0.45 & nd & nd & nd & nd & nd & nd \\
\hline 3 & Verbenene & 0.99 & nd & nd & nd & nd & nd & nd \\
\hline 4 & Sabinene & $\mathrm{nmt}$ & 0.8 & 0.8 & 0.8 & 0.8 & $\mathrm{nmt}$ & nd \\
\hline 5 & $\beta$-pinene & $\mathrm{nmt}$ & 23.7 & 16.7 & 25.6 & 18.4 & 6.3 & 18.76 \\
\hline 6 & $\alpha$-Phellandrene & 12.01 & 0.6 & nd & $\mathrm{nmt}$ & $\mathrm{nmt}$ & $\mathrm{nmt}$ & nd \\
\hline 7 & $\alpha$-Terpinene & 1.24 & nd & nd & $\mathrm{nmt}$ & $\mathrm{nmt}$ & nd & nd \\
\hline 8 & Limonene & 0.72 & nd & nd & nd & nd & $\mathrm{nmt}$ & nd \\
\hline 9 & $\beta$-Phellandrene & $\mathrm{nmt}$ & 1.0 & $\mathrm{nmt}$ & 0.5 & $\mathrm{nmt}$ & nd & nd \\
\hline 10 & $\gamma$-Terpinene & 23.22 & 17.6 & 11.8 & 20.2 & 20.1 & 6.1 & 16.86 \\
\hline 11 & p-Cymene & 15.87 & 13.7 & 4.9 & 6.0 & 8.0 & 17.4 & 0.30 \\
\hline 12 & p-Menth-2-en-1-ol & 0.80 & nd & nd & nd & nd & nd & nd \\
\hline 13 & $\begin{array}{l}\text { 2-Methyl-4- } \\
\text { isopropyliden- } \\
\text { cyclopentan-1-al }\end{array}$ & 0.54 & nd & nd & nd & nd & nd & nd \\
\hline 14 & (-)-4-Terpineol & 0.54 & nd & nd & $\mathrm{nmt}$ & $\mathrm{nmt}$ & $\mathrm{nmt}$ & 2.44 \\
\hline 15 & trans-Dihydrocarvone & 31.11 & nd & nd & nd & nd & nd & nd \\
\hline 16 & p-Cuminaldehyde & 0.58 & 36.1 & 26.2 & 19.5 & 23.6 & 49.4 & 36.67 \\
\hline 17 & $\alpha$-Thujene & nd & $\mathrm{nmt}$ & $\mathrm{nmt}$ & $\mathrm{nmt}$ & $\mathrm{nmt}$ & $\mathrm{nmt}$ & 1.88 \\
\hline 18 & p-Menth-2-en-7-ol & 3.48 & nd & nd & nd & nd & nd & nd \\
\hline 19 & Teresantalol & 2.62 & nd & nd & nd & nd & nd & nd \\
\hline 20 & $\alpha$-terpinen-7-al & nd & 1.9 & 6.8 & 5.1 & 7.5 & 6.8 & nd \\
\hline 21 & $\gamma$-Terpinen-7-al & nd & 2.4 & 30.2 & 29.2 & 17.8 & nd & nd \\
\hline 22 & p-cymen-7-ol & $\mathrm{nmt}$ & nd & nd & nd & nd & 4.6 & nd \\
\hline 23 & Thymol & nd & nd & nd & nd & nd & 2.8 & 15.48 \\
\hline 24 & Carvacrol & nd & nd & nd & nd & nd & $\mathrm{nmt}$ & 10.72 \\
\hline
\end{tabular}

*: - samples were collected form the local market, **: - samples were collected from local market of Serbia but the samples originated from India, nmt: - Not more than $0.5 \%$, nd: - not detected Table no. 3 chemical composition of cumin oil from Cuminum cyminum seeds. Compounds more than $0.5 \%$ are complied.
Jiang from China reported cuminaldehyde along with cuminic alcohol, $\gamma$-Terpinene, safranal, $\mathrm{p}$-cymene and $\beta$ Pinene as the main chemical components of the cumin oil. Pakistan's cumin oil showed presence of terpenes in the order of cuminyl alcohol $>$ cuminaldehyde $>\beta$-Pinene $>$ p-cymene $>\alpha$-Terpinene $>1,8$-Cineole. Muna F. Abushama et al reported 2-Caren-10-al, cumialdehyde, $\gamma$-Terpinene, $\beta$ Pinene, $\mathrm{p}$-cymene and $\alpha$-Pinene as six main compounds of Sudan's cumin oil.

Cumin oil from different regions of India showed presence of p-cymene, a natural antioxidant, from $\min 0.30 \%$ - $\max$ $17.4 \%$, cuminaldehyde in the range of $0.58 \%-49.4 \%$ and $\gamma$ Terpinene $(6.1 \%-23.22 \%)$. Along with these compounds, $\beta$ pinene, $\alpha$-pinene, $\alpha$-terpinen-7-al and $\gamma$-Terpinen-7-al were

The data tabulated in table 3 shows the main compounds identified and detected in the cumin oil extracted from cumin seeds which were collected from different regions of India. also reported.

\section{CONCLUSION}

In the present study, cumin oil was extracted from cumin seeds by hydro distillation. The extracted cumin oil and market cumin oil showed a major difference in the chemical 
composition. But the main component i.e. cuminaldehyde was found in extracted as well as market cumin oil. The manufacturer has not mentioned the extraction procedure; hence conclusion cannot be drawn. $\alpha$-Neocallitropsene which was detected only in market cumin oil while it was not found to be present in any other research papers, it can be concluded that the market cumin oil might be contaminated with $\alpha$-Neocallitropsene. $\alpha$-Neocallitropsene is a unique and main chemical component of Eryngium duriaei $[25,26]$.

The comparative data of the chemical composition of the cumin oil from different countries revealed that the composition of the oil significantly depends on number of factors. Factors that influence the production and composition of essential oils in include (a) physiological variations (b) environmental conditions (c) geographic variations (d) genetic factors and evolution (e) political/social conditions and also (f) amount of plant material/ space and manual labour needs. There are innumerable examples of the occurrence of geographic variations of the yield and composition of volatiles. The different essential oil compositions of a species found for different origins reflect the different environmental conditions of each particular location and culture conditions (different altitudes, different solar exposition, different soil types, etc.) [27].

Mohamed Elsaed Ebada mentioned in his review paper the various medical and non-medical benefits of cuminaldehyde. He reported in his review paper that cuminaldehyde has been recently found to exert protective effects against neurodegenerative diseases in particular Parkinson's disease. This was evidenced by its long-lasting suppressing effects on the fibrillation of alpha synuclein, the pathogenesis hallmark of Parkinson's disease, compared to baicalein, a standard alpha synuclein fibrillation suppressor. Additional investigation suggested that cuminaldehyde impaired $\beta$ structural assembly of alpha synuclein fibrils, thereby inhibiting its fibrillation [28]. Ramin Rezaee, Hossein Hosseinzadeh reported safranal medical benefits as anticonvulsant in their review article. Safranal was reported to have medical benefits like anticonvulsant, cerebral ischemia, antidepressant etc [29]. Hence it can be concluded that cumin seeds collected in the present study which showed a high amount of cuminaldehdye can be used for neurodegenerative diseases. Similarly, cumin seeds from Iran, with a good amount of safranal, should be included in the diet for anticonvulsant benefits.

\section{Acknowledgment}

The authors are grateful and express their sincere regards to Dr. Ganapathy Ramakrishnan, Hon-Director SIES-ICS, Mr. Dipak Shetty, Laboratory Manager, SIES-ICS and Ms.
Sneha Nair, Laboratory Chemist SIES-ICS for providing a friendly environment and research facilities.

\section{References}

[1] E. V. D. Sastry and M. Anandaraj, Soils, plant growth and crop production - Cumin, Fennel and Fenugreek, Encyclopedia of Life Support Systems (EOLSS).

[2] F. H. Y. Al-Hashemi, Chromatographic separation and identification of some volatile oils, organic acids and phenols from the seeds of Cuminum cyminum growing in Iraq, International Journal of Research and Reviews in Applied Sciences, Vol. 19, Issue 1, pp- 80-90, 2014.

[3] N. Chaudhary, S. S. Husain, M. Ali, Chemical composition and antimicrobial activity of volatile oil of the seeds of Cuminum cyminum 1., World Journal of Pharmacy and Pharmaceutical Sciences, Volume 3, Issue 7, pp 1428-1441, 2014.

[4] M. G. Aćimović, V. Tešević, D. Mara, M. Cvetković, J. Stanković, V. Filipović, The analysis of cumin seeds essential oil and total polyphenols from postdestillation waste material, Advanced Technologies, Volume 5, Issue 1, pp 23-30, 2016.

[5] D. Kaur, R. Sharma, An Update on Pharmacological Properties of Cumin, International Journal of Research in Pharmacy and Science, Volume 2, Issue 4, pp 14-27, 2012.

[6] N. Chaudhary, S. S. Husain, M. Ali, New Phenolic, triterpenic and steroidal constituents from the fruits of Cuminum cyminum L., Journal of Pharmacognosy and Phytochemistry, Volume 3, Issue 1, pp 149-154, 2014.

[7] S. Pandey, M. K. Patel, A. Mishra, B. Jha, Physio-Biochemical Composition and Untargeted Metabolomics of Cumin (Cuminum cyminum L.) Make It Promising Functional Food and Help in Mitigating Salinity Stress, Plos one, Volume 10, Issue 12, pp 1$25,2015$.

[8] Deepak, Importance of Cuminum cyminum L. and Carum Carvi L. in traditional medicaments - a review, Indian Journal Traditional Knowledge, Volume 12, Issue 2, pp 300-307, 2013.

[9] V. A. Parthasarathy, B. Chempakam and T. J. Zachariah, Chemistry of Spices, Biddles Ltd, King's Lynn, ISBN 978-1-84593-405-7, pp 211-226, 2008.

[10] K. V. Peter, Handbook of herbs and spices, Woodhead Publishing Limited, England, pp 164-167, 2001.

[11] S. A. Moawad, A. H. El-Ghorab, M. Hassan, H. Nour-Eldin, M. M. El-Gharabli, Chemical and Microbiological Characterization of Egyptian Cultivars for Some Spices and Herbs Commonly Exported Abroad, Food and Nutrition Sciences, Volume 6, pp 643-659, 2015.

[12] L. Gachkar, D. Yadegari, M. B. Rezaei, M. Taghizadeh, S. A. Astaneh, I. Rasooli, Chemical and biological characteristics of Cuminum cyminum and Rosmarinus officinalis essential oils, Food Chemistry, Volume 102, Issue 3, pp 898-904, 2007.

[13] H. Mohammadpour, E. Moghimipour, I. Rasooli, M. H. Fakoor, S. A. Astaneh, S. S. Moosaie, Z. Jalili, Chemical Composition and Antifungal activity of Cuminum cyminum L. essential oil from Alborz mountain against Aspergillus species, Jundishapur Journal of Natural Pharmaceutical Products, Volume 7, Issue 2, pp 50-55, 2012.

[14] A. Naeini and H. Shokri, Chemical composition and in vitro antifungal activity of the essential oil from Cuminum cyminum against various Aspergillus strains, Journal of Medicinal Plants Research Volume 6, Issue 9, pp. 1702-1706, 2012.

[15] R. Li and Z.-T. Jiang, Chemical composition of the essential oil of Cuminum cyminum L. from China, Flavour and Fragrance Journal, Volume 19, pp 311-313, 2004.

[16] N. Hashemian, A. G. Pirbalouti, M. Hashemi, A. Golparvar, B. Hamedi, Diversity in chemical composition and antibacterial 
activity of essential oils of cumin (Cuminum cyminum L.) diverse from northeast of Iran, Australian Journal of Crop Science, Volume 7, Issue 11, pp 1752-1760, 2013.

[17] A. R. L. Moghadam, Essential oil of the seeds of Cuminum cyminum L.(Apiaceae), Bulletin of Environment, Pharmacology and Life Sciences, Volume 4, Issue 3, pp 161-163, 2015.

[18] D. Morshedi, T. Salmani Kesejini, F. Aliakbari, R. Karami-Osboo, M. Shakibaei, A. Tayaranian Marvian, M. Khalifeh and M. Soroosh, Identification and characterization of a compound from Cuminum cyminum essential oil with antifibrilation and cytotoxic effect, Research in Pharmaceutical Sciences, Volume 9, Issue 6, pp 431-443, 2014.

[19] Z. Ghafari, A. Alizadeh, R. B. Samani, Antimicrobial activity and essential oil composition of Cuminum cyminum L. and Carum carvi L. seeds from Iran, International Journal of Biosciences, Volume 4, Issue no. 6, pp- 153-159, 2014.

[20] A. H. Chaudhry, A. Tanveer, A. Shar, M. S. Akhtar, M. K. Shahid, K. M. Ashfaq, T. A. Malik and R. ul H. Siddiqui, PhysicoChemical Investigation and Antimicrobial Activity of Essential Oil of Cuminum cyminum L., World Applied Sciences Journal, Volume 19, Issue 3, pp 330-333, 2012.

[21] A. R. Pour, S. S. Mirzargar, M. Soltani, H. A. E. Mousavi and S. A. Mostafavi, The antibacterial effects of Cuminum cyminum $L$. and Rosmarinus officinalis extracts and essential oil against Lactococcus garvieae in laboratory conditions on rainbow trout, European Journal of Experimental Biology, Volume 4, Issue 1, pp 456-463, 2014.

[22] M. F. Abushama, H. Yasmin, H. Abdalgadir and H. Khalid, Chemical Composition, Antimicrobial and Brine Shrimp Lethality of the Essential Oil of Cuminum cyminum L., International Journal of Pharmaceutical and Chemical Sciences, Volume 2, Issue 4, pp 1666-1672, 2013.

[23] V. S. Rana, Chemical Composition of the Essential Oil of Cuminum cyminum L. Seeds from Western India, Journal of Medicinal Plants and By-products, Volume 2, pp 207-210, 2014.

[24] S. D. Patil, P. P. Maknikar, S. J. Wankhade, C. S. Ukesh, M. K. Rai, Chemical composition, antimicrobial and antioxidant activity of essential oils from cumin and ajowan, Nusant ara bioscience, Volume 8, Issue 1, pp. 60-65, 2016.

[25] A. C. Tavares, A. D. Rodrigues, A. L. Soares, C. P. Gomes, C. Castel-Branco, D. Batista, G. Luckhurst, I. Fontes, M. C. Castro, M. Freire, M. M. Silva, M. P. Alonso, M. C. de Sousa, J. Nunes, R. Carvalho, R. Sá Correia, R. S. Matos, T. Chambel, The Garden as a Lab Where Cultural and Ecological systems meet in the Mediterranean Context, 2014, Centro de História da Arte e Investigação Artística of the Universidade de Évora, ISBN: 978989-99083-3-8, Chaia, pp 148-162, 2014.

[26] T. Nuzhat and G. M. Vidyasagar, Antifungal investigations on plant essential oils. a Review, International Journal of Pharmacy and Pharmaceutical Sciences, Volume 5, Suppl 2, pp 19-28, 2013.

[27] Priya ES, Selvan PS and Yavanarajan A, Chemotypic variation in Clove oil and Lemongrass oil obtained from three different geographical locations of India, Pharmacognosy and Natural Products, Volume 1, Issue 1, PP 1-4, 2015.

[28] M. E. Ebada, Cuminaldehye: A potential drug candidate, Journal of Pharmacology and Clinical Research, Volume 2, Issue 2, pp 14, 2017.

[29] R. Rezaee and H. Hosseinzadeh, Safranal: From an aromatic natural product to a rewarding pharmacological agent, Iranian Journal of basic medical sciences, Volume 16, Issue 1, pp 12-26, 2016.

[30] S. Kapoor and N. Sarwate, Effects of indigenous foods (Fenugreek, black sesame and bitter cumin) on blood glucose level of type-2 diabetes patients, International Journal of Scientific Research in Biological Sciences, Volume 2, Issue 5, pp. 17-20, 2015.

\section{AUTHORS PROFILE}

Esha Tambe pursued M.Sc. in Analytical Chemistry from Institute of Science in 2011. She is currently pursuing Ph.D. in Analytical Chemistry since 2016 from SNDT Women's University under the guidance of Mrs. Sulekha Gotmare. The area of interest of research is essential oil, especially spice oils. She is a Life Member of Chromatographic Society of India. She had published 4 papers including one review paper on Rose oil in International Journals. Recently she was awarded with Best Paper Award at ICNFESMH 2018 held at Pune on $17^{\text {th }}$ June, 2018.

Sulekha Gotmare pursued $\mathrm{Ph} . \mathrm{D}$ in Analytical Chemistry. She is currently Head In charge and an Associate Professor of Department of Analytical Chemistry at S.H.P.T College of Science, SNDT Women's University, Santacruz, India. She has more than 10 publications. Areas of Interest of research are cosmetology, food analysis, pharmaceutical analysis, etc. 